\title{
Pathological characteristics and prognostic effect of peritumoral capsule penetration in renal cell carcinoma after tumor enucleation
}

\author{
Andrea Minervini, M.D., Ph.D. ${ }^{\mathrm{a}, *}$, Maria Rosaria Raspollini, M.D., Ph.D. ${ }^{\mathrm{b}}$, \\ Agostino Tuccio, M.D. ${ }^{a}$, Claudio Di Cristofano, M.D. ${ }^{c}$, Giampaolo Siena, M.D. , \\ Matteo Salvi, M.D. ${ }^{a}$, Gianni Vittori, M.D. ${ }^{\mathrm{a}}$, Arcangelo Sebastianelli, M.D. ${ }^{\mathrm{a}}$, \\ Alberto Lapini, M.D. ${ }^{\text {, }}$ Sergio Serni, M.D. ${ }^{a}$, Marco Carini, M.D. ${ }^{\text {a }}$ \\ ${ }^{\text {a }}$ Department of Urology, University of Florence, Careggi Hospital, Florence, Italy \\ ${ }^{\mathrm{b}}$ Department of Pathology, University of Florence, Careggi Hospital, Florence, Italy \\ ${ }^{\mathrm{c}}$ Department of Experimental Medicine, Sapienza University of Rome, Polo Pontino, I.C.O.T, Latina, Italy
}

Received 12 May 2013; received in revised form 29 July 2013; accepted 30 July 2013

\begin{abstract}
Objective: To evaluate the pathological characteristics of peritumoral capsule (PC) and the prognostic effect of capsule penetration on tumor recurrence in patients treated with tumor enucleation for clinically intracapsular renal cell carcinomas (RCCs).

Methods and materials: PC status was analyzed in 304 consecutive patients with single intracapsular RCC. Degree and side of capsule penetration if present were evaluated. Mean (median, range) follow-up was 49 months (46, 25-69). Local recurrence rate, progression-free survival (PFS), and cancer-specific survival were the main outcomes. Statistical analyses included the Kaplan-Meier method, log-rank test, and univariate and multivariate Cox regression models.

Results: Overall, 51\% of RCCs had intact PC and free from neoplastic invasion (PC-), 34.9\% had capsular penetration on the parenchymal side (PCK), and $14.1 \%$ had tumor invasion on the perirenal fat tissue side (PCF). None of the patients had positive surgical margins. The 5-year PFS rates for tumors PC-, PCK, and PCF were 97.5\%, 96.7\%, and 77.1\%, respectively $(P<0.0001)$.

The multivariate Cox model showed PCF to be the sole significant independent predictor of PFS, whereas patients who had PCK did not present a significant increased risk in developing recurrence.

Conclusions: Tumor enucleation is an oncologically safe nephron-sparing surgery technique. PCF is a significant and independent predictor of tumor recurrence in patients with clinically intracapsular RCCs scheduled for nephron-sparing surgery. PCK does not predict the risk of recurrence. (C) 2014 Elsevier Inc. All rights reserved.
\end{abstract}

Keywords: Capsular invasion; Peritumoral capsule; Prognosis; Renal cell carcinoma; Tumor enucleation; Partial nephrectomy

\section{Introduction}

With the widespread use of modern imaging techniques, a growing number of small renal tumors are being diagnosed and treated by nephron-sparing surgery (NSS). The recent European Association of Urology guidelines expanded its indication for solitary renal cell carcinoma (RCC) up to a diameter of $7 \mathrm{~cm}$, whenever technically feasible. A minimal tumor-free surgical margin appears appropriate to avoid the risk of local recurrence, thus

\footnotetext{
* Corresponding author. Tel.: +39-055-41-7645; fax: +39-055-437-7755.

E-mail addresses: andreamine@libero.it, andrea.minervini@unifi.it (A. Minervini).
}

supporting the possible use of the tumor enucleation (TE), which is a nephron-sparing procedure with oncological effectiveness supported by numerous papers (Level of Evidence 2b) [1-8]. Over the past years, this technique has received a wider consensus as an alternative to standard partial nephrectomy for T1 RCC and has been adopted in several centers [8].

Overall, many large series with single sporadic RCC treated conservatively by either standard partial nephrectomy or TE reported favorable survival and recurrence rates, but tumor progression may occur in some cases [2,4-7,9-12]. This evidence has led to identifying clinical and pathological parameters as predictors of disease progression. The available published evidence indicates that the TNM 
staging system, tumor size, histological subtype, Fuhrman grade, and indications for NSS (elective vs. imperative) are significant predictors of cancer-specific survival (CSS) [12-15].

The TNM staging system uses tumor size as a single prognostic factor for clinically intracapsular RCC. However, tumor size may be considered a scarcely reliable prognostic factor in distinguishing tumors with different biological behavior, and other pathological parameters could bring a significant improvement in the prognostic assessment of RCCs.

Most renal tumors amenable for conservative surgery tend to compress the normal parenchyma and have a continuous fibrous capsule around it [3,16-19]. We hypothesized that peritumoral fibrous capsule penetration on either the parenchymal side or the perinephric adipose tissue side might provide the first pathological evidence of tumor cells' capacity to infiltrate and invade surrounding tissue, which could eventually increase the risk of tumor recurrence, thus emerging as a useful and significant prognostic factor in patients with clinically intracapsular RCC [3,16-19]. The prognostic implication of renal capsular involvement in patients with RCC is quite well assessed [18-25]. However, until now there is no knowledge about the correlation of the individual oncological course with extend and side of infiltration of the tumor capsule. The aim of the present study is to analyze prospectively the pathological characteristics, to examine the side and degree of capsular penetration in patients with single, sporadic, clinically intracapsular RCC, and to evaluate the prognostic effect of capsule penetration on local and systemic recurrence in a consecutive series of 304 patients treated by TE.

\section{Methods and materials}

After institutional review board approval was obtained, data were gathered prospectively from 451 consecutive patients who underwent TE between January 2005 and June 2011. Patients with histologically confirmed benign tumors (97 patients, $21.5 \%$ ), patients with solitary kidney owing to previous RCC treated by radical nephrectomy (19 patients, $4.2 \%$ ), and those with multiple ipsilateral (22 patients, $4.9 \%$ ) and synchronous bilateral RCCs (9 patient, 2\%) were excluded from the study, thus leaving 304 (67.4\%) consecutive patients treated with elective NSS (277 patients, $91.2 \%$ ) or with imperative indication (27 patients, $8.8 \%$ ). The preoperative evaluation included abdominal ultrasonography and computed tomography (CT) with contrast medium and a chest x-ray in all patients. None of the 304 patients had preoperative or intraoperative suspicion of positive nodes. All patients were presumably free from distant metastases before surgery (M0).

In all cases, TE was done according to our previously described standard protocol, by blunt dissection with no visible rim of healthy tissue and always using the natural cleavage plane between the tumor and normal parenchyma $[3,5]$. The tumor specimen was oriented in the operating theater, positioning one suture at the deepest part of the inner pole of the tumor and another, of a different color, at the level where the tumor became exophytic.

After formalin fixation (10\%), all specimens were stepsectioned at 5-mm intervals, and the entire specimen was analyzed by 2 dedicated uropathologists. Patients were staged according to the 2009 American Joint Committee on Cancer-Union International Centre le Cancer TNM classification [26], and nuclear grading was assigned according to criteria proposed by Fuhrman et al. [27]. The histopathology was reviewed according to the 2004 WHO classification [28]. The existence, integrity, width, and depth of cancer penetration of peritumoral capsule (PC), if present, were evaluated and recorded. PC thickness was measured at the inner and at the outer pole of the specimen. PC classification is reported in the Panel (Fig. 1).

The follow-up schedule included blood chemistries and chest X-rays in association with either ultrasound or CT of the abdomen every 3 to 4 months for the first year after surgery, every 6 months from postoperative years 2 to 5 , and annually thereafter. Bone scintigraphy and chest CT were used only when there was clinical suspicion.

The unpaired student $t$ test and $\chi^{2}$ test were used to evaluate the possible statistical correlation between the clinical and pathological variables and the risk of PC invasion. Local recurrence rate, progression-free survival (PFS), and CSS were the main outcomes of this study. The probability of survival was estimated by the Kaplan-Meier method, with the log-rank test used to estimate differences among levels of the analyzed variables. Univariate and multivariate Cox regression models addressed time to PFS. TNM stage was not included in the multivariable model to avoid the risk to overfit the model because of colinearity with $\mathrm{PC}$ status (perirenal fat tissue side $[\mathrm{PCF} H]$ equal to focal pT3a). Statistical significance was set at $P \leq 0.05$. Analyses were conducted using all events in Stat View 5.0.1 (SAS Institute Inc., Cary, NC).

\section{Results}

Preoperative characteristics, clinical presentation, and pathological data of the 304 patients included in the study are reported in Table 1.

All RCCs were surrounded by a continuous, not fenestrated fibrous capsule constituted by dense connective fibrous tissue. Mean PC thickness at the inner pole of the tumor (standard deviation, median, and range) was $412 \mu \mathrm{m}$ $(250,350,20-1,511)$. Mean PC thickness at the outer pole of the tumor (standard deviation, median, and range) was $385 \mu \mathrm{m}(253,358$, and 20-1,770, respectively). Of the $304 \mathrm{RCCs}$, in 155 (51\%), the peritumoral capsule was intact and free from neoplastic invasion ( $\mathrm{PC}-$ ), whereas in 

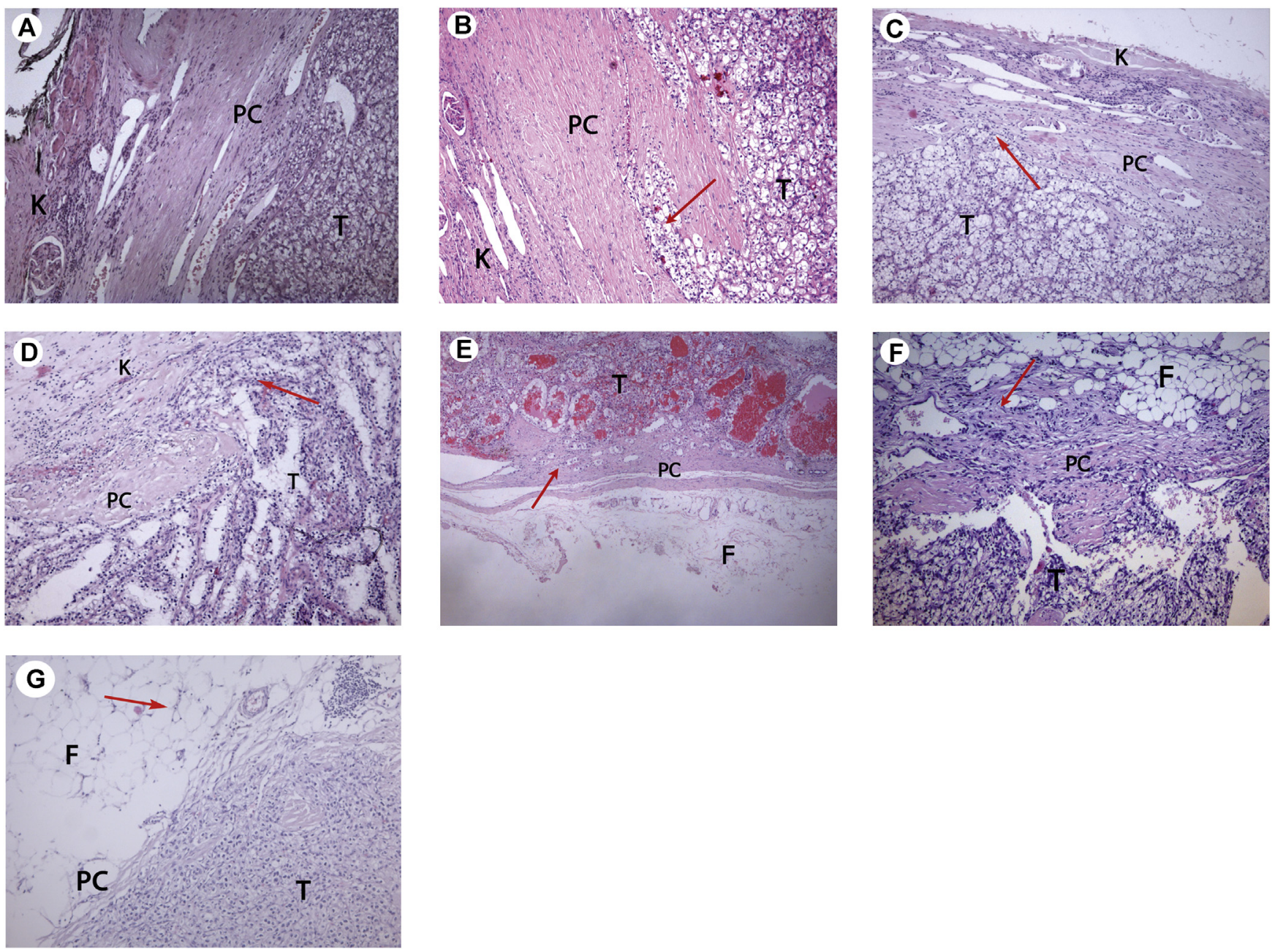

Fig. 1. (A) $\mathrm{PC}-(\mathrm{PC}$ intact and free from neoplastic penetration); (B and $\mathrm{C}) \mathrm{PCK}+(\mathrm{PC}$ with signs of neoplastic penetration on parenchymal kidney side); (D) $\mathrm{PCK}+$ (PC with complete neoplastic penetration on parenchymal kidney side); (E) PCF+ (PC with signs of neoplastic penetration on the perirenal adipose tissue side); (F and G) PCF+ (PC with complete neoplastic penetration on the adipose tissue side, focal microscopic pT3a). K = kidney; PC = peritumoral capsule; $\mathrm{T}=$ tumor; $\mathrm{F}=$ perirenal fat. Red arrow indicates neoplastic penetration. (Color version of figure is available online.)

149 (49\%), there were signs of penetration within its layers. The capsular features according to the side and degree of neoplastic penetration are reported in Fig. 1 and Table 2. The pathologists applied ink to the enucleated tumor in all cases, but in none the ink touched the surface of the tumor.

Correlation analyses showed a statistically significant association of capsular invasion with symptoms at presentation, TNM stage, and tumor greatest dimension, whereas age, imperative indications for surgery, histological subtype, Fuhrman grade, and PC thickness were not.

Mean (median, range, and interquartile range) follow-up was 49 months $(52,12-96$, and 16-88, respectively). During follow-up, 13 patients had progressive disease (4.3\%). Clinicopathological characteristics and outcomes of patients who had disease progression are reported in Table 3.

The 5-year overall survival was 93.8\%. The 3- and 5-year CSS rates were 98.2\%. The 3- and 5-year PFS rates were $95.3 \%$ and $94.8 \%$, respectively. The crude recurrence rate according to PC status was $1.9 \%$ for $\mathrm{PC}-, 1.3 \%$ for $\mathrm{PCK}+$, $6.4 \%$ for PCK+, $11.1 \%$ for PCF+, and $20 \%$ for PCF + . The 5-year PFS rate for RCC according to PC status was as follows: PC-, 97.5\%; PCK+, 98.2\%; PCK+, 92.8\%; PCF+, 82.6\%; and PCF+, $74 \%(P<0.0001$; $\mathrm{PC}-$ vs. PCK,$+ P=$ 0.68 ; PC- vs. PCKH, $P=0.1247$; $\mathrm{PC}-$ vs. PCF+, $P<0.0113$; and $\mathrm{PC}-$ vs. PCF $4, P<0.0001$; Fig. 2).

No statistically significant difference in the risk of progression was observed between PCK + and PCKH $(P=$ $0.106)$ and between PCF + and PCF $+(P=0.6)$. Therefore, we defined 3 prognostic classes, i.e. $\mathrm{PC}-$, PCK $(+$ and +$)$, and PCF $(+$ and + ). The 5-year PFS rates for tumors PC-, PCK, and PCF were $97.5 \%, 96.7 \%$, and $77.1 \%$, respectively $(P<0.0001)$. At univariate analysis, factors associated with worse PFS were capsular penetration on the perirenal tissue side, imperative indications for conservative surgery, tumor greatest dimension, and higher tumor stage (Table 4). The TNM stage was not included in the multivariable model because of colinearity with PC status, and multivariable Cox regression model showed PCF to be the sole significant 
Table 1

Patients' characteristics, clinical presentation, and pathological features of 304 renal cell carcinomas (RCCs) treated with NSS

\begin{tabular}{|c|c|}
\hline Variables & $\begin{array}{l}n(\%) \text { or mean } \\
(\mathrm{SD} ; \text { range; IQR) }\end{array}$ \\
\hline Mean age & $63( \pm 13 ; 22-85 ; 56-73)$ \\
\hline \multicolumn{2}{|l|}{ Gender } \\
\hline Male & $192(63 \%)$ \\
\hline Female & $112(37 \%)$ \\
\hline \multicolumn{2}{|l|}{ Tumor side } \\
\hline Right & $164(53.9 \%)$ \\
\hline Left & $140(46.1 \%)$ \\
\hline No. Symptomatic at presentation & $30(9.8 \%)$ \\
\hline \multicolumn{2}{|l|}{ Indications for NSS } \\
\hline Elective & $277(91.2 \%)$ \\
\hline Imperative & $27(8.8 \%)$ \\
\hline \multicolumn{2}{|l|}{ Tumor location } \\
\hline Upper pole & $79(26 \%)$ \\
\hline Mid kidney & $136(44.7 \%)$ \\
\hline Lowe pole & $71(23.3 \%)$ \\
\hline Perihilar & $18(6 \%)$ \\
\hline Mean (SD; range) clinical tumor size $(\mathrm{cm})$ & $3.4(1.4 ; 1.2-10)$ \\
\hline Mean (SD; range) pathological tumor size $(\mathrm{cm})$ & $3.4(1.4,1-12.5)$ \\
\hline \multicolumn{2}{|l|}{ Tumor histotype } \\
\hline Clear cell RCC & $209(68.8 \%)$ \\
\hline Papillary RCC & $53(17.5 \%)$ \\
\hline Chromophobe RCC & $39(12.8 \%)$ \\
\hline Others & $3(0.9 \%)$ \\
\hline \multicolumn{2}{|l|}{ TNM 2009} \\
\hline pT1a & $215(70.7 \%)$ \\
\hline pT1b & $60(19.8 \%)$ \\
\hline $\mathrm{pT} 2 \mathrm{a}$ & $3(0.9 \%)$ \\
\hline $\mathrm{pT} 2 \mathrm{~b}$ & $1(0.3 \%)$ \\
\hline pT3a & $25(8.2 \%)$ \\
\hline \multicolumn{2}{|l|}{ Fuhrman grade } \\
\hline G1 & $42(15.5 \%)$ \\
\hline G2 & $201(74.2 \%)$ \\
\hline G3 & $27(10 \%)$ \\
\hline G4 & $1(0.3 \%)$ \\
\hline
\end{tabular}

$\mathrm{IQR}=$ interquartile range; $\mathrm{SD}=$ standard deviation

independent predictor of PFS (Table 4). In particular, patients with PCF had a 6-fold higher risk of disease progression than patients with an intact capsule (confidence interval [CI] 1.579-29.085; $P=0.01$ ) and a 5-fold higher risk of disease progression than PCK (CI 1.244-21.220; $P=0.0237$ ), whereas patients who had PCK did not present a significant increased risk in developing a relapse in comparison with $\mathrm{PC}-(\mathrm{CI} 0.262-6.649 ; P=0.7371$; Table 4).

Then, we created a subset of patients by eliminating $\mathrm{PCF}+$ and $\mathrm{PCK}+$ with the aim to confirm the prognostic role of capsular invasion also in the subgroups of tumors with no tumor cells outside the peritumoral capsule. At univariate Cox model, $\mathrm{PCF}+$ was confirmed to be significantly associated with a worse PFS $(P=0.0421)$ (Table 5).

\section{Discussion}

The studies on the capsular characteristics and involvement in RCCs were historically focused on the parenchymal side of the tumor to evaluate the technical feasibility and oncological safety of blunt TE, and a higher rate of PC invasion in larger and less differentiated tumors was reported [29]. More recently, Li et al. [18] prospectively studied 82 kidneys with RCC with a mean tumor diameter of $3.4 \mathrm{~cm}$ and showed a capsular penetration on the kidney side in $26.8 \%$ of cases, which was confirmed by us in a previous study (26.6\%) and was even higher in the present consecutive series (34.9\%) [3].

The evaluation of capsular involvement on the perirenal tissue side is more recent with the aim to better stratify the prognosis of patients with intracapsular tumors. These studies on large retrospective series showed a great variability in $\mathrm{PC}$ invasion rate on the perirenal tissue side $(\mathrm{PCF}+)(7.2 \%-37.5 \%$, Table 6) [20-25]. In our series of RCC with a mean (range) tumor size of $3.4 \mathrm{~cm}$ (1-12.5), $\mathrm{PCF}+$ was present in $5.9 \%$ of RCCs, similar to the $7.2 \%$ reported by Rouach and coworkers in a study of 305 RCCs with a mean (range) tumor dimension of $3.5(0.5-15) \mathrm{cm}$. One possible explanation to this great variability is the difference in mean tumor dimension but also in the reported incidence of clear cell RCC, although ourselves and Klatte et al. [23] did not find an association between tumor histotype and PC invasion [3]. Another possible reason is related to the different magnifying resolution possibly used to define PC invasion between studies that eventually led us to diagnose a higher rate of $\mathrm{PCF}+(8.2 \%$ of focal microscopic pT3a), but unfortunately these data were not reported in most of the studies on this topic [20-25]. Indeed, a

Table 2

PC features of 304 renal cell carcinomas (RCCs) from patients who underwent tumor enucleation

\begin{tabular}{|c|c|c|c|c|c|c|}
\hline PC status & Number of cases $(\%)$ & $\%$ & Clear cell $(\%)$ & Papillary (\%) & Chromophobe $(\%)$ & Others $(\%)$ \\
\hline $\mathrm{PC}-$ & 155 & 51 & $110(52.6)$ & $23(43.4)$ & $19(48.7)$ & $3(100)$ \\
\hline PCK & 106 & 34.9 & $71(34)$ & $19(35.8)$ & $16(41)$ & 0 \\
\hline PCK+ & 75 & 24.7 & $54(25.9)$ & $7(13.2)$ & $14(35.9)$ & 0 \\
\hline $\mathrm{PCK}+$ & 31 & 10.2 & $17(8.1)$ & $12(22.6)$ & $2(5.1)$ & 0 \\
\hline PCF & 43 & 14.1 & $28(13.4)$ & $11(20.8)$ & $4(10.3)$ & 0 \\
\hline $\mathrm{PCF}+$ & 18 & 5.9 & $11(5.3)$ & $4(7.6)$ & $3(7.7)$ & 0 \\
\hline $\mathrm{PCF}+$ & 25 & 8.2 & $17(8.1)$ & $7(13.2)$ & $1(2.6)$ & 0 \\
\hline Total & 304 & 100 & 209 & 53 & 39 & 3 \\
\hline
\end{tabular}




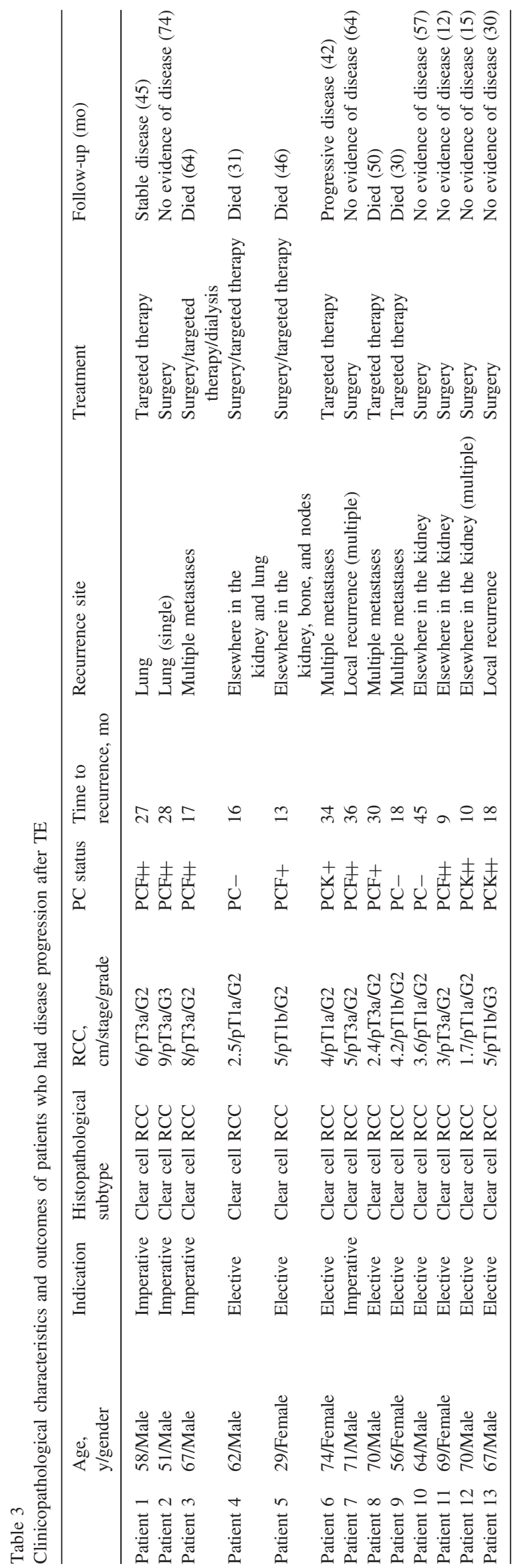

standardized method to analyze the PC is necessary to exclude this bias from future studies.

Peritumoral capsule pathological analysis and its evaluation as a prognostic factor are not new for pathologists. Indeed, in other tumors, as in follicular thyroid neoplasm, the penetration of fibrous capsule was analyzed and was found to be associated with a malignant phenotype [30]. Also for RCCs, the presence of a capsular involvement could represent the first pathological evidence of the capacity achieved by tumor cells to infiltrate and invade normal parenchyma and perirenal tissue, and might potentially increase the risk of local and systemic recurrence, and could eventually be used as a prognostic factor in patients with clinically intracapsular RCC amenable for conservative surgery. However, the prognostic effect of peritumoral fibrous capsule penetration remains controversial [3,1925]. A few authors recently investigated the prognostic implication of capsular involvement on the perinephric fat side in patients treated with either radical nephrectomy or NSS (Table 6) [20-25]. Overall, more than 2,000 patients were evaluated, and the mean follow-up ranged between 40 and 86 months [20-25]. Two reports did not find PCF+ to be a predictor of disease recurrence [24,25]. Possible criticisms of these papers reside in their retrospective nature, the long time frame of the studies, and the reevaluation of selected slides only, instead of the entire specimen. On the contrary, most of these reports showed $\mathrm{PCF}+$ to have a significant effect on disease-free survival [20-24]. In our series, we confirm the prognostic effect of $\mathrm{PCF}+$ at univariate analyses, and we did not observe a statistically significant difference in the risk of progression between PCF+ and PCF + , in agreement with Klatte et al. [23] who reported PCF+ tumors having an identical recurrence-free survival to those with $\mathrm{PCF}+\mathrm{H}$. In our analysis, we merged PCF+ and PCF+t and showed that this neoplastic event (PCF) was the only independent predictor of recurrence-free survival. In patients with clinically intracapsular RCCs scheduled for NSS, capsular invasion on the perinephric tissue side appears to be a strong predictor of recurrence-free survival (Table 4). Moreover, our accurate pathological analysis allowed us to conclude that RCCs suitable for NSS are surrounded by a continuous, not fenestrated fibrous pseudocapsule and that $\mathrm{PCK}+$ and PCK+ do not present a significant increased risk of developing local and systemic recurrence in comparison with PC- when TE is performed, and it clearly represents a rationale to safely adopt this technique for the excision of clinically intracapsular RCC. Peritumoral pseudocapsule can be penetrated irrespective of tumor size, with a reported infiltration rate of $34.9 \%$ on the parenchymal side, but the presence of a thin layer of parenchymal tissue with signs of lymphoplasmocytic inflammation allows for negative surgical margins, also if no efforts are made to leave a rim of healthy kidney tissue around the neoplasm [3]. This thin rim of normal parenchymal tissue is always present in case of neoplastic penetration of the 


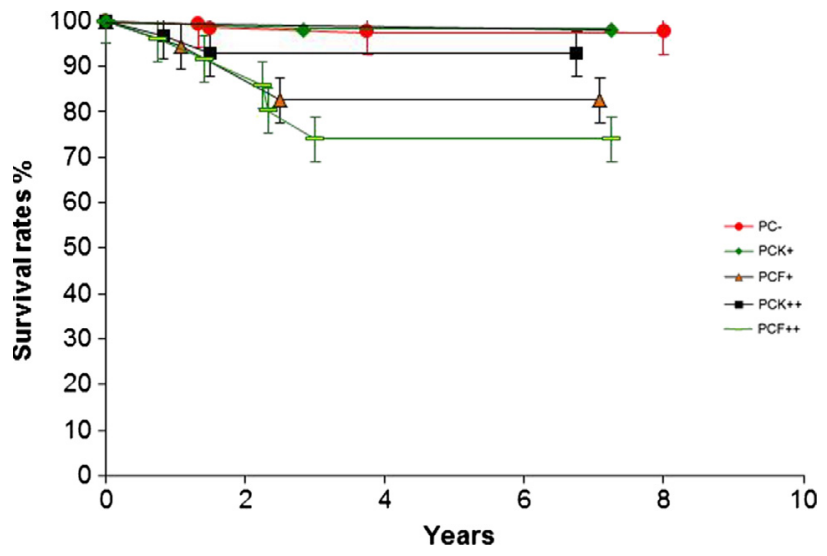

\begin{tabular}{|lcccccc|}
\hline Years & 1 & 2 & 3 & 4 & 5 & \\
Pts at risk & 153 & 127 & 104 & 77 & 39 & PC- \\
& 74 & 65 & 55 & 51 & 39 & PCK+ \\
& 17 & 10 & 5 & 5 & 4 & PCF+ \\
& 29 & 23 & 14 & 12 & 8 & PCK++ \\
& 24 & 17 & 12 & 11 & 9 & PCF++ \\
& & & & & & \\
\hline
\end{tabular}

Fig. 2. Progression-free survival (PFS) probability according to PC status. Five-year PFS was $97.5 \%$ in PC-, $98.2 \%$ in $\mathrm{PCK}+, 92.8 \%$ in PCKH, $\mathrm{PCF}+, 82.6 \%$ in $\mathrm{PCF}+$, and $74 \%$ in $\mathrm{PCF}+$. (Color version of figure is available online.)

pseudocapsule into the kidney tissue (PCK+H). Therefore, if the surgeon follows the natural cleavage plane between tumor pseudocapsule and kidney parenchyma by blunt dissection, thus performing a TE, there is a limited risk of positive surgical margins even with larger masses.
Table 5

Univariable Cox regression analysis for prediction of progression-free survival in the subgroup of tumors with no tumor cells outside the peritumoral capsule (245 patients; $\mathrm{PC}-, \mathrm{PCK}+$, and $\mathrm{PCF}+$ )

\begin{tabular}{llll}
\hline Parameter & \multicolumn{3}{l}{ Univariable analysis } \\
\cline { 2 - 4 } & HR & $95 \%$ CI & $P$ value \\
\hline Pathological tumor size, continuous & 1.156 & $0.740-1.805$ & 0.5240 \\
Pathological T stage & & & 0.4347 \\
T1a & 1 & Reference & - \\
T1b & 1.967 & $0.360-10.749$ & - \\
PC status & & & 0.0421 \\
PC- & 1 & Reference & - \\
PCK+ & 1.569 & $0.163-15.103$ & 0.6965 \\
PCF+ & 12.421 & $1.105-139.577$ & 0.0412 \\
\hline
\end{tabular}

$\mathrm{HR}=$ hazard ratio

In daily pathology practice, addressing the peritumoral capsule status is easy and straightforward without the need for obtaining special stains or extra slides and without the additional expense in time and cost, and according to the results obtained by our study and by previous retrospective series, we believe that this feature should be routinely assessed during the specimen analysis $[3,19]$.

Limitations of the present study include the relatively few patients enrolled, which however exceeds that of most peer articles, and the medium-term follow-up, as systemic tumor recurrence after NSS has been reported to occur well past the 49-month follow-up horizon. However, this is a homogeneous series of patients, all had NSS performed by TE, and 2 dedicated uropathologists thoroughly analyzed the entire specimens and completed the pathological reports in consensus with a precise pathological analysis. Further

Table 4

Univariable and multivariable Cox regression analyses for prediction of progression-free survival in 304 patients after TE

\begin{tabular}{|c|c|c|c|c|c|c|}
\hline \multirow[t]{2}{*}{ Parameter } & \multicolumn{3}{|c|}{ Univariable analysis } & \multicolumn{3}{|c|}{ Multivariable analysis } \\
\hline & HR & $95 \% \mathrm{CI}$ & $P$ value & HR & $95 \% \mathrm{CI}$ & $P$ value \\
\hline Age, continuous & 0.990 & $0.949-1.033$ & 0.6434 & - & - & - \\
\hline Symptoms at presentation & & & 0.3586 & - & - & - \\
\hline Absent & 1 & Reference & - & & & \\
\hline Present & 2.030 & $0.448-9.198$ & - & & & \\
\hline Indication for surgery & & & 0.0101 & & & 0.2324 \\
\hline Elective & 1 & Reference & - & 1 & Reference & - \\
\hline Imperative & 4.704 & $1.446-15.303$ & - & 2.224 & $0.599-8.256$ & - \\
\hline Pathological tumor size, continuous & 1.333 & $1.084-1.638$ & 0.0064 & 1.169 & $0.885-1.544$ & 0.2701 \\
\hline Pathological $\mathrm{T}$ stage & & & 0.0015 & - & - & - \\
\hline $\mathrm{T} 1 \mathrm{a}$ & 1 & Reference & - & & & \\
\hline $\mathrm{T} 1 \mathrm{~b}$ & 2.064 & $0.493-8.641$ & 0.3211 & & & \\
\hline $\mathrm{T} 3 \mathrm{a}$ & 9.531 & $2.756-32.959$ & 0.0004 & & & \\
\hline Fuhrman nuclear grade & & & 0.4523 & - & - & - \\
\hline G1-2 & 1 & Reference & - & & & \\
\hline G3-4 & 1.783 & $0.394-8.064$ & - & & & \\
\hline PC status & & & 0.0006 & & & 0.0133 \\
\hline $\mathrm{PC}-$ & 1 & Reference & - & 1 & Reference & - \\
\hline PCK & 1.438 & $0.290-7.125$ & 0.6566 & 1.319 & $0.262-6.649$ & 0.7371 \\
\hline $\mathrm{PCF}$ & 10.095 & $2.601-39.180$ & 0.0008 & 6.777 & $1.579-29.085$ & 0.0100 \\
\hline
\end{tabular}

$\mathrm{HR}=$ hazard ratio. 


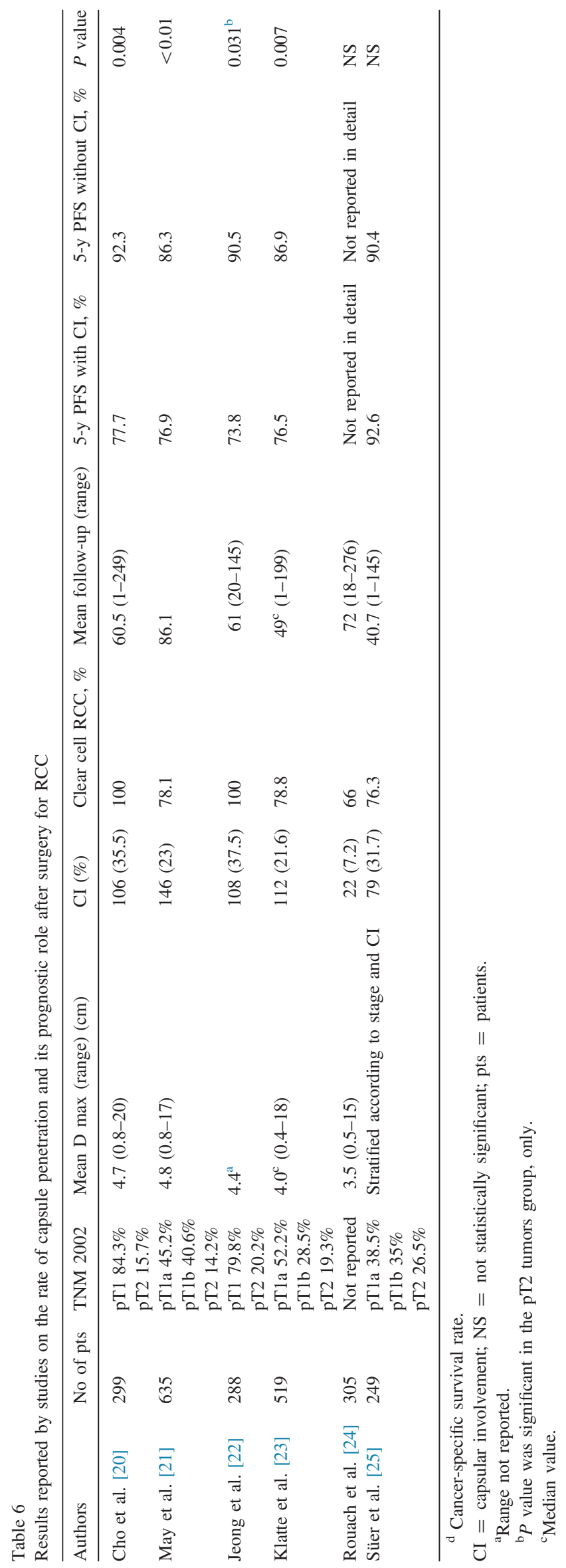

advantage of this work is assessment based on a standardized prospective protocol of histopathological evaluation of the specimens. However, even based on a standardized protocol with $5 \mathrm{~mm}$ step-sections, some areas of the peritumoral capsule remained unrated.

We acknowledge that a number of our pathological findings on a limited number of patients have already appeared in the literature [3,5]. However, this study represents the first series where capsular involvement on both the perinephric side and the parenchymal side was evaluated from a prognostic perspective at a mean followup of more than 4 years and reported not only the rate of local recurrence but also systemic relapses.

Additional observation would be mandatory and could eventually reinforce our results, and multicentric studies should be conducted to strengthen the statistical power of the study.

\section{Conclusions}

Peritumoral capsule invasion is a frequent finding in localized RCCs, which appears to occur at a higher rate on the parenchymal side than on the perirenal tissue side in the reported cases. A capsule penetration on the perirenal adipose tissue side is a significant and independent predictor of tumor recurrence, with a relative risk of disease progression nearly 6 times higher than an intact peritumoral capsule. For clinically intracapsular tumors amenable for conservative surgery, the accurate analysis of the capsular status at the perirenal tissue side could overtake the TNM staging system for risk stratification of disease recurrence. A capsule penetration on the parenchymal side does not predict the risk of local and systemic recurrence even if a TE is performed.

\section{References}

[1] Ljungberg B, Cowan NC, Hanbury DC, et al. EAU guidelines on renal cell carcinoma: the 2010 update. Eur Urol 2010;58:398-406.

[2] Becker F, Siemer S, Humke U, et al. Elective nephron sparing surgery should become standard treatment for small unilateral renal cell carcinoma: long-term survival data of 216 patients. Eur Urol 2006;49:308-13.

[3] Minervini A, Di Cristofano C, Lapini A, et al. Histopathological analysis of peritumoral pseudocapsule and surgical margins status after tumor enucleation for renal cell carcinoma. Eur Urol 2009;55: $1410-8$.

[4] Kutikov A, VanArsdalen KN, Gershman B, et al. Enucleation of renal cell carcinoma with ablation of the tumour base. BJU Int 2008;102: 688-91.

[5] Minervini A, Serni S, Tuccio A, et al. Local recurrence after tumour enucleation for renal cell carcinoma with no ablation of the tumour bed: results of a prospective single-centre study. BJU Int 2011;107:1394-9.

[6] Minervini A, Ficarra V, Rocco F, et al. Simple enucleation is equivalent to traditional partial nephrectomy for renal cell carcinoma: results of a nonrandomized, retrospective, comparative study. J Urol 2011;185:1604-10.

[7] Minervini A, Serni S, Tuccio A, et al. Simple enucleation versus radical nephrectomy in the treatment of pT1a and pT1b renal cell carcinoma. Ann Surg Oncol 2012;19:694-700. 
[8] Laryngakis NA, Guzzo TJ. Tumor enucleation for small renal masses. Curr Opin Urol 2012;22:365-71.

[9] Patard JJ, Shvarts O, Lam JS, et al. Safety and efficacy of partial nephrectomy for all $\mathrm{T} 1$ tumors based on an international multicenter experience. J Urol 2004;171:2181-5.

[10] Leibovich BC, Blute ML, Cheville JC, et al. Nephron sparing surgery for appropriately selected renal cell carcinoma between 4 and $7 \mathrm{~cm}$ results in outcome similar to radical nephrectomy. J Urol 2004;171: 1066-70.

[11] Mitchell RE, Gilbert SM, Murphy AM, et al. Partial nephrectomy and radical nephrectomy offer similar cancer outcomes in renal cortical tumors $4 \mathrm{~cm}$ or larger. Urology 2006;67:260-4.

[12] Fergany AF, Hafez KS, Novick AC. Long-term results of nephron sparing surgery for localized renal cell carcinoma: 10-year follow up. J Urol 2000;163:442-5.

[13] Krejci KG, Blute ML, Cheville JC, et al. Nephron-sparing surgery for renal cell carcinoma: clinicopathologic features predictive of patient outcome. Urology 2003;62:641-6.

[14] Pahernik S, Roos F, Wiesner C, et al. Nephron sparing surgery for renal cell carcinoma in a solitary kidney. World J Urol 2007;25:513-7.

[15] Ghavamian R, Cheville JC, Lohse CM, et al. Renal cell carcinoma in the solitary kidney: an analysis of complications and outcome after nephron sparing surgery. J Urol 2002;168:454-9.

[16] Vermooten V. Indications for conservative surgery in certain renal tumors: a study based on the growth pattern of the clear cell carcinoma. J Urol 1950;64:200.

[17] Minervini A, Tuccio A, Lapini,, et al. Review of the current status of tumor enucleation for renal cell carcinoma. Arch Ital Urol Androl 2009;81:65-71.

[18] Li Q-L, Guan H-W, Zhang Q-P, et al. Optimal margin in nephronsparing surgery for renal cell carcinoma $4 \mathrm{~cm}$ or less. Eur Urol 2003;44:448-51.

[19] Di Cristofano C, Minervini A, Tuccio A, et al. Pathological evaluation of peritumoral pseudocapsule status after nephron sparing surgery and its prognostic implication. Arch Ital Urol Androl 2009;81:96-9.

[20] Cho H-J, Kim SJ, Ha U-S, et al. Prognostic value of capsular invasion for localized clear-cell renal cell carcinoma. Eur Urol 2009;56:1006-12.

[21] May M, Brookman-Amissah S, Roigas J, et al. Evaluation of renicapsular involvement in stages I and II renal cell carcinoma from the morphological and prognostic point of view. Urol Oncol 2010;28:274-9.

[22] Jeong IG, Jeong CW, Hong SK, et al. Prognostic implication of capsular invasion without perinephric fat infiltration in localized renal cell carcinoma. Urology 2006;67:709-12.

[23] Klatte T, Chung JS, Leppert JT, et al. Prognostic relevance of capsular involvement and collecting system invasion in stage I and II renal cell carcinoma. BJU Int 2007;99:821-4.

[24] Rouach Y, Delongchamps N, Timsit M-O, et al. Capsular involvement in patients undergoing partial nephrectomy for localized renal cell carcinoma: an adverse pathological finding? BJU Int 2010;105:616-9.

[25] Süer E, Ergün G, Baltaci S, et al. Does renal capsular invasion have any prognostic value in localized renal cell carcinoma? J Urol 2008;180:68-71.

[26] Sobin LH, Gospodariwicz M, Wittekind C, editors. TNM Classification of Malignant Tumors. UICC International Union Against Cancer, 7th ed. Wiley-Blackwell: 2009. Available at: http://www.uicc.org/tnm.

[27] Fuhrman SA, Lasky LC, Limas C. Prognostic significance of morphologic parameters in renal cell carcinoma. Am J Surg Pathol 1982;6:655-63.

[28] Lopez-Beltran A, Scarpelli M, Montironi R, et al. 2004 WHO classification of the renal tumors of the adults. Eur Urol 2006;49: 798-805.

[29] Rosenthal CL, Kraft R, Zingg EJ. Organ-preserving surgery in renal cell carcinoma: tumor enucleation versus partial kidney resection. Eur Urol 1984;10:222-8.

[30] Evans HL. Follicular neoplasms of the thyroid. A study of 44 cases followed for a minimum of 10 years, with emphasis on differential diagnosis. Cancer 1984;54:535-40. 